\title{
COSMOLOGICAL EVIDENCE FROM \\ QSOS AND RADIO GALAXIES
}

\author{
M. J. REES
}

Institute of Theoretical Astronomy, Cambridge, Great Britain

\begin{abstract}
Cosmological evidence provided by counts of radio and optical objects, and additional evidence yielded by QSO redshifts, are discussed. The nature of the QSO redshifts is considered, as are the evolutionary properties of these and other objects, and possibly important cosmological investigations using QSOs and radio sources are mentioned.
\end{abstract}

\section{Introduction}

Attempts to use observations of ordinary galaxies to discriminate between different cosmological models have already been reviewed by Dr Peach. He showed that these are as yet somewhat inconclusive, basically because galaxies become invisibly faint before one gets to the redshifts where substantial deviations from the linear Hubble law are expected. In this talk I shall try to summarize what has been learnt from objects which may be detectable at greater distances, namely the strong radio sources and the QSOs. It turns out, unfortunately, that these studies have by no means clarified the situation. Indeed they are no help at all in deciding which - if any - of the 'standard' cosmological models best describes our universe, and they fail even to provide a useful estimate of the deceleration parameter $q_{0}$. The reasons for this regrettable situation are twofold. First, there is a huge spread in the intrinsic luminosity of radio sources (and in the optical luminosity of QSOs, if their redshifts are cosmological). Therefore no 'standard candles' are available, so one must fall back on crude statistical tests. Second, our physical understanding of these objects is so limited that we have no idea what evolutionary corrections to apply.

However the data do already imply something which is surely crucially important for the astrophysics of compact objects and 'violent events': namely that whichever of the standard cosmologies we choose, there must be strong secular evolution in the coordinate density of strong radio sources and QSOs, in the sense that there were many more powerful objects at earlier cosmic epochs.

I shall discuss first (Section 2) the general cosmological evidence provided by counts, and then (Section 3) the additional information yielded by the redshifts of the QSOs. (At this stage it is of course necessary to adopt some hypotheses regarding the nature of QSO redshifts; some relevant arguments are considered later in a separate section (Section 5)). I shall then discuss more fully the character of the strong source evolution which the data indicate, and whether it is plausible on astrophysical grounds (Section 4). The extent to which unconventional hypotheses may remove the need to invoke this evolution is also briefly considered. Finally (Section 6), I shall enumerate some other potentially important cosmological tests involving QSOs and radio sources. 


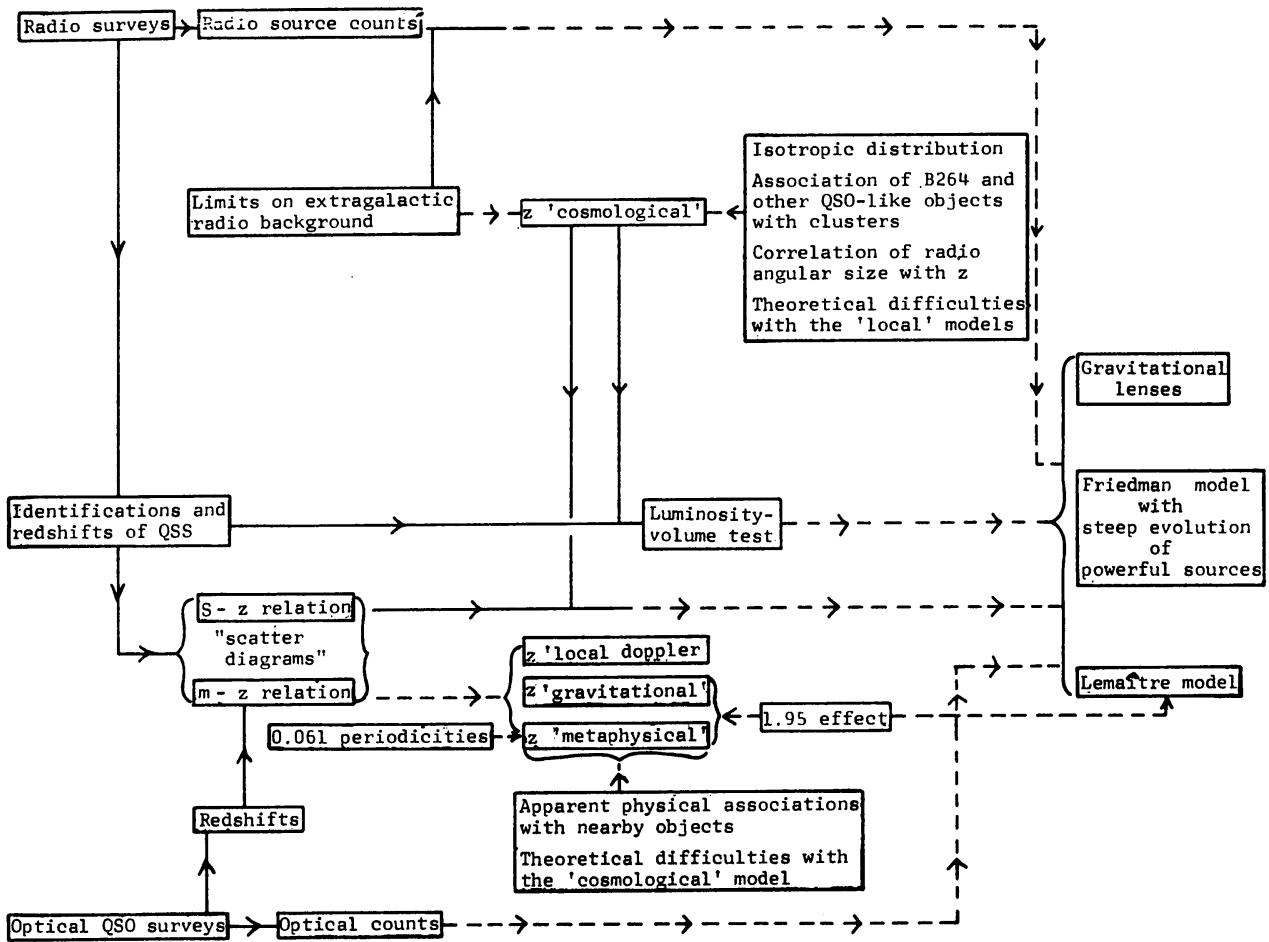

Fig. 1. Schema for QSO and QSS research.

Figure 1 is an attempt to summarise how various observations of QSOs and radio galaxies pertain to cosmology. Possible inferences are indicated by dashed arrows, but one should certainly not attach equal weight to them all - they range from the fairly plausible to the exceedingly dubious.

\section{Radio (and Optical) Counts}

\section{A. RADIO EVIDENCE}

The identified radio sources span an extremely broad range of both luminosity and linear dimensions, and no type of source has yet been found which serves as an adequate 'standard candle'. As a consequence, the most fruitful cosmological test utilizing radio data alone has been comparison of the relative numbers of strong and faint sources revealed by radio surveys. The conventional procedure is to plot, against flux density $S$, the number $N$ of sources per steradian brighter than $S$. To get good statistics at high flux densities, where the number of sources is small, one needs a survey covering a large area of sky; however a smaller area suffices at lower flux levels (provided that the Universe is sufficiently isotropic and homogeneous).

Source counts have now been performed by many different groups working independently, and when allowance is made for statistical uncertainty, and for the different 
frequencies at which the surveys were made, there is good agreement among all the results obtained. I shall first mention the counts presented by Pooley and Ryle (1968), which provide good statistics over the widest range of flux density and have formed the framework for many theoretical investigations. These counts are based on the $5 \mathrm{C} 2$ survey, covering a few square degrees of sky down to a flux level $S_{408} \simeq 10^{-2} \mathrm{f}$.u., together with the earlier $3 \mathrm{C}$ and $4 \mathrm{C}$ surveys. Source counts based on data from the $3 \mathrm{C}$ and $4 \mathrm{C}$ surveys, which were carried out at $178 \mathrm{MHz}$, had previously been constructed by Gower (1966)*. If all the sources had identical power-law spectra, Gower's curve could have been straightforwardly scaled to $408 \mathrm{MHz}$, and matched onto the $N / S$ curve from the deep 5C survey. However, because there is a spread in the spectral indices, this simple procedure is illegitimate. Instead, Pooley and Ryle had to redetermine the flux of a large sample of the brighter sources at $408 \mathrm{MHz}$. For 3C sources, the spectra were in general sufficiently well established to do this by interpolation, but it was necessary to carry out a separate fan beam survey (Bailey and Pooley, 1968) to determine $408 \mathrm{MHz}$ fluxes for a sample of $4 \mathrm{C}$ sources.

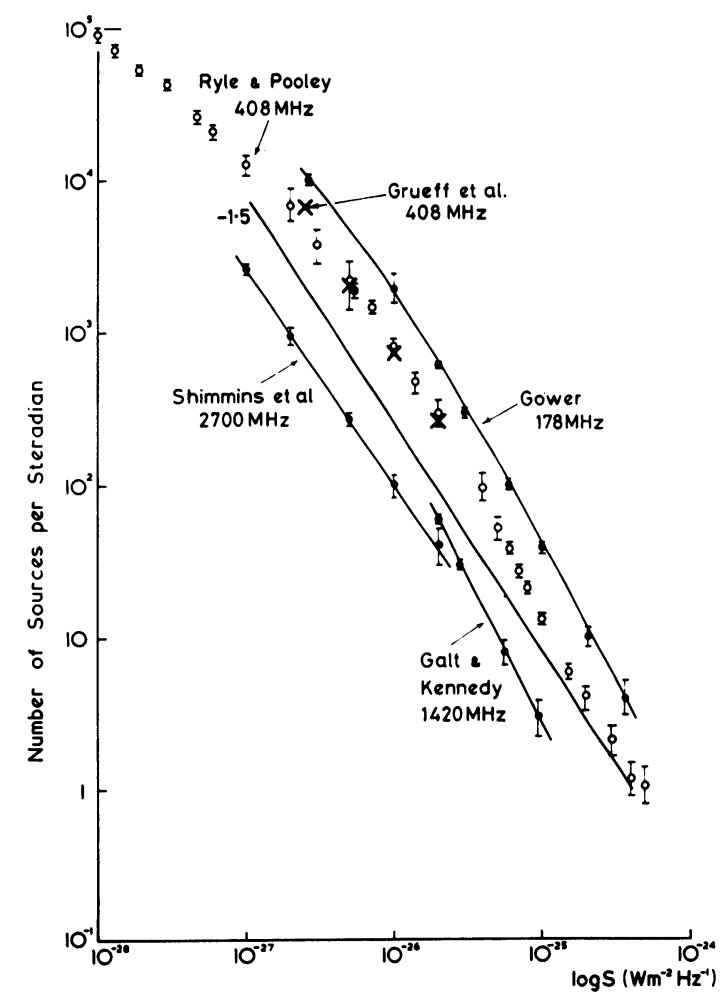

Fig. 2. The source counts from Pooley and Ryle (1968). Also shown are the earlier Cambridge counts at $178 \mathrm{MHz}$, due to Gower (1966), together with counts from some recent higher-frequency surveys mentioned in the text. (reproduced from Shakeshaft (1968))

* Gower's curve actually extended down to values of $S_{408}$ a factor 8 below the $4 \mathrm{C}$ limit, the lower flux part being based on data from the North Polar survey (Ryle and Neville, 1962). 
Pooley and Ryle's results, plotted on the traditional form $\log N$ vs $\log S$, are shown in Figure 2. The high flux (3C) part of the graph is approximately linear, with a slope of $\sim-1.8$, but there is a gradual flattening towards the fainter end, and at $S_{408} \simeq 0.01$ the slope is only $\sim-0.8$. (Of course, the curve must eventually flatten to a slope $>-1$ to ensure convergence of the integrated radio background).

Independent surveys by Bolton et al. (1964); MacLeod et al. (1965); Braccesi et al. (1966); and Grueff and Vigotti (1968) yield source counts which are entirely consistent with the Cambridge results. (The first of these provided comforting assurance that the overall structure of the Universe appears the same from the Southern Hemisphere.)

More recently, several surveys have been carried out at much higher frequencies than $408 \mathrm{MHz}$. In comparing the results of these with the earlier work it is important to remember that, because there is a spread in the spectral indices of radio sources, one does not expect precisely the same $N(S)$ relation at all frequencies. High frequency surveys are weighted in favour of sources with flat or rising spectra, and there is no obvious reason why these should have the same space distribution as the type of source which predominates in the Cambridge surveys (and in the other comparatively low frequency surveys mentioned above). In principle, one can predict the $N(S)$ relation at a frequency $v_{2}$ in terms of the counts at a different frequency $v_{1}$ if one knows the spectral index of all the sources. However if the spectral indices are widely spread one needs to know $N(S)$ at $v_{1}$ down to extremely low fluxes in order to predict even the bright end of the $N(S)$ relation at $v_{2}$ - for example, some of the brightest sources in a $5 \mathrm{GHz}$ survey have rising spectra, and would be below even the $5 \mathrm{C}$ cutoff at $408 \mathrm{MHz}$.

The recent Ohio survey at $1415 \mathrm{MHz}$ (Harris and Kraus, 1970) covers a large enough area of sky to provide good statistics at the low- $N$ end of the $\log N / \log S$ curve which plays the most crucial role in the interpretations. The quoted slope over this part of the curve is -1.7 , essentially the same as the 3C slope. Galt and Kennedy (1968) had earlier surveyed a smaller area at a similar frequency. Shimmins et al. (1968) at Parkes, carried out a $2700 \mathrm{MHz}$ survey covering 0.35 ster. down to $S_{2700}=0.4 \mathrm{f}$.u. together with a deeper survey, covering a smaller area, which extended down to a limiting flux of $0.08 \mathrm{f}$.u. The quoted $\log N / \log S$ slope was $-1.4 \pm 0.1$. In relating two $N(S)$ relations, one should of course compare the form of the curves at corresponding values of $N$, and these Parkes counts should be compared with the intermediate (4C) segment of the Cambridge curve. The slopes then agree well (Pooley, 1968). This survey did not cover a large enough area of sky to provide good statistics on the ' $3 C$ ' segment $\left(N \lesssim 100\right.$ ster $\left.^{-1}\right)$. There are indications that the $2700 \mathrm{MHz}$ counts fail to steepen, perhaps because of a few bright sources with flat spectra absent from the 3C survey, but it is not clear how significant this is (van der Laan, 1969; Wall, 1970). A $5000 \mathrm{MHz}$ survey carried out at N.R.A.O. (Kellermann et al., 1968; Pauliny-Toth et al., 1971) finds an $N(S)$ relation which fully agrees with what one would expect on the basis of lower frequency data.

In evaluating the reliability and significance of any $N(S)$ relations, a number of 
possible sources of error must be considered. (These matters are further discussed by Ryle (1968); Scheuer (1970); and in the original papers on the various surveys.) Obviously it is essential that the flux estimates be reliable over the whole range of $S$, and that the survey be complete. The effects of confusion tend to cause the fainter sources to be systematically overestimated in flux. Moreover, any random uncertainties in the fluxes of individual sources can cause systematic errors in the $N(S)$ relation. Another problem is that surveys made using interferometers fail to register the full flux of sources with large angular size. It is possible to estimate how important such effects are likely to be, and (to some extent) to correct for them in the final $N(S)$ curve. However all these effects can still contribute to the total errors, especially at low flux levels. Even if no errors or uncertainties of any kind entered into the observational procedure, there would still be a 'formal error' attached to the $N(S)$ curve, and to the quoted slope, because of the finite number of sources involved. (We may envisage this error as an estimate of the variations in the $N(S)$ relation that would be observed by an ensemble of observers scattered through space.) This type of 'error' constitutes the main uncertainty in the $3 \mathrm{C}$ part of the Cambridge survey.

It is very important that, taking into account the different populations sampled at different frequencies, all the counts are mutually compatible. What, then, does the $N(S)$ relation tell us? The first basic point is that in a static Euclidean universe a uniform distribution of sources would yield a curve $N \propto S^{-3 / 2}$ (i.e. a $\log N / \log S$ slope of -1.5) irrespective of the luminosity function.*

The expected $N(S)$ curve can be calculated for a steady-state expanding universe, and also for the standard Friedman models, on the assumption that the comoving density of each type of source is the same at all epochs. The detailed formulae are derived in Scheuer's (1970) review. The spectral index of the sources now enters explicitly, but the key result is that, at least in models where the cosmical constant $\Lambda$ is zero, any uniformly distributed population of sources whose spectra fall towards higher frequency yields a $\log N / \log S$ slope flatter than -1.5.** (Lemaître-type cosmologies will be further discussed in Section 4). The precise predicted slope obviously depends on the redshifts of the sources making the main contribution at a particular value of $S$, and so involves the luminosity function $\varrho(P)$.

The crucial feature of the Cambridge counts is the steep slope of the $3 \mathrm{C}$ part of the $N(S)$ curve. Assuming that the 'error' results only from the finite number of sources,

\footnotetext{
* The unwary might be tempted to argue, as follows, that a luminosity function such that $Q(P)$ rises steeply with decreasing $P$ could yield a steep slope: each spherical shell around the observer makes a contribution to the $\log N / \log S$ curve that reflects the slope of the luminosity function (and which may therefore be steeper than -1.5), and so, if the luminosity function is broad enough, the steep slope will be preserved when we integrate the contributions from all shells. But the argument is invalid because, for a sufficiently steep luminosity function (and one in fact would require $Q(P) \mathrm{d} P$ steeper than $P^{-5 / 2} \mathrm{~d} P$ ) the main contribution to the high- $S$ part of the counts comes from intrinsically faint sources in nearby shells which would not contain even one bright source. Allowing for these sources, one still recovers the -1.5 slope unless there is a 'local hole' surrounding the observer.

** If the sources have power-law spectra with $S(v) \propto v^{-\alpha}$, then the effects of the ' $K$-correction' steepen the $N(S)$ above its Euclidean value only if $\alpha<-1$ (Einstein-de Sitter) or $\alpha<-2.5$ (steady state).
} 
the slope differs from 1.5 at the $3 \sigma$ level.* However, both the steady state model and 'source conserving' Friedman models would give rise to substantially flatter slopes, because a large fraction of the $3 \mathrm{C}$ sources have substantial redshifts (this is true even if we exclude QSS and confine attention to the radio galaxies whose redshifts are agreed to be 'cosmological' in origin). This greatly increases the significance of the counts, since the disparity between the observations and the non-evolutionary predictions extends down to lower fluxes, where the numbers involved are larger and the statistics consequently better.

Non-evolutionary models are unable to reproduce this highly significant feature of the counts, and there have consequently been many explorations - mainly in the framework of standard Friedman cosmologies - of the form of this evolution. I shall now outline the general conclusions that can be drawn from the radio data alone, but defer detailed consideration of the character of the evolution until the optical evidence from QSOs has been discussed (in Section 3).

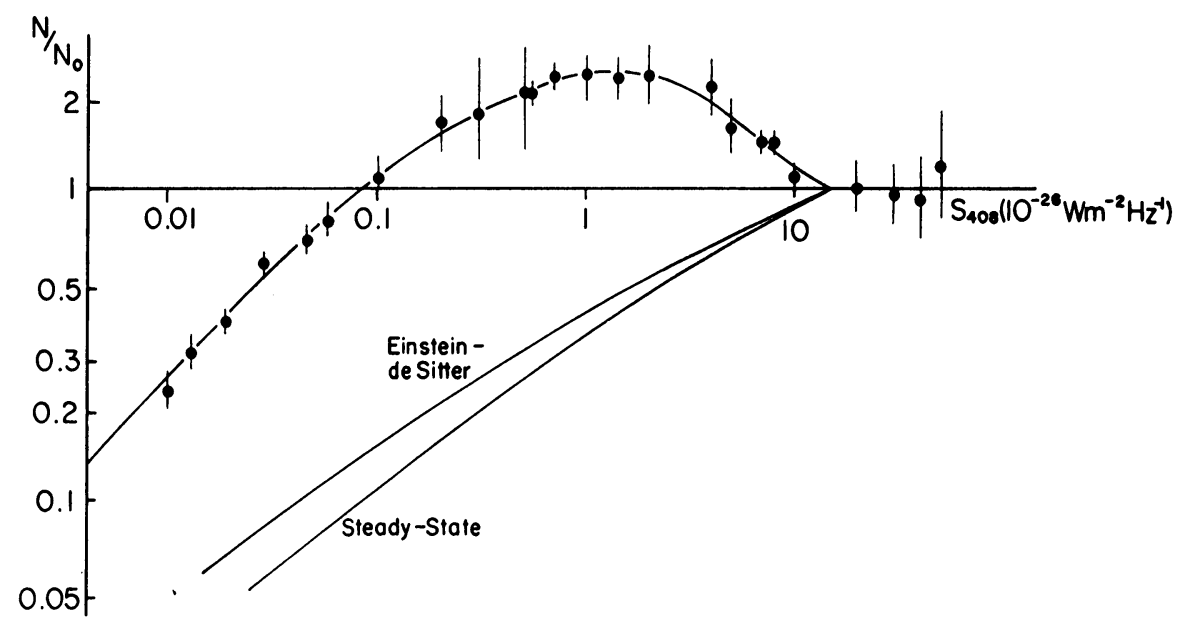

Fig. 3. Pooley and Ryle's counts plotted in terms of $N / N_{0}$ (see text for explanation). The other curves show the expected forms of the curve in Steady State and Einstein-de Sitter cosmologies, if the sources had the same luminosity function and the same coordinate density at all redshifts. (reproduced from Ryle (1968))

The nature of the problem can perhaps be clarified by displaying the data somewhat differently. Figure 3 shows the same counts as Figure 2 from the Cambridge surveys plotted in terms of $N / N_{0}$, where $N_{0}$ is the number of sources with flux density $>S$

* In estimating the slope and its uncertainty, it is important to remember that successive points in an integral $N(S)$ curve are not independent. There are consequently some advantages in plotting counts differentially (Jauncey, 1967; Refsdal, 1969). By fitting a 'maximum likelihood' straight line to the $3 C$ differential $\log N / \log S$ curve, Jauncey derived a slope $2.79(+0.12,-0.11)$. (But there is of course no reason to expect any part of the curve to be a precise straight line.) Longair and Scheuer (1970) show that the significance of source counts can also be estimated by exploiting their close relationship to the luminosity-volume test. 
expected in a Euclidean universe, where the $\log N / \log S$ relation would always follow a straight line with slope -1.5 . ( $N$ is normalised to $N_{0}$ at $\sim 10 \mathrm{ster}^{-1}$, about the smallest value at which the statistics are significant). In a 'source conserving' cosmology - whether Einstein-de Sitter or steady state $-N / N_{0}$ would decrease monotonically towards smaller values of $S$, and the slope of the expected curves, for the observationally determined luminosity function, is indicated in Figure 3. The observed $N / N_{0}$, however, rises markedly above unity at intermediate fluxes (the disparity being most significant at $S_{408} \simeq 2$ f.u.), and falls away sharply at the faint end. The 'excess' of intermediate flux sources amounts to at least $\sim 1000 \mathrm{ster}^{-1}$ (and is vastly greater if one compares with the predictions of a source-conserving expanding universe instead of a Euclidean model); however one can equally legitimately think in terms of a deficit of bright sources, in which case the numbers involved are at least $\sim 30 \mathrm{ster}^{-1}$. $^{\dagger}$

Once one admits the possibility that the comoving density of each type of source (and hence the luminosity function $\varrho(P)$ ) can change with epoch, the 'excess' shown in Figure 3 can be attributed to a population at $z \dot{\sim} z^{*}$ (say) whose nearby counterparts (which would tend to raise the high- $S$ part of the curve, thus destroying the excess) are absent, or at least have a much lower density per comoving volume element. Obviously one can fit the observed peak in the $N / N_{0}$ diagram equally well by evolving $\varrho(P)$ with $z$ in an enormous variety of ways. There are, however, some additional general constraints that must be satisfied. The sharp drop at low flux levels must be explained, as well as the excess. Also, the best estimates of the extragalactic radio background indicate that about half is accounted for by sources bright enough to be counted individually ${ }^{\dagger \dagger}$, and a smooth extrapolation of Pooley and Ryle's curve could account for the remaining half, so this tells us that $N / N_{0}$ must continue to fall steeply at fluxes below the $5 \mathrm{C}$ limit.

Two conclusions follow from these constraints.

(i) It is implausible that $z^{*} \ll 1$, because in any Friedman-type model the sources with a given $P$ at redshifts in the range $z^{*}$ to $\sim 1$ would span a range of $\sim z^{*-2}$ in observed flux. Since the effects of the redshift do not drastically flatten the $N(S)$ curve until $z$ becomes $\sim 1$, the peak in Figure 3 would be too broad, even if all the sources had exactly the same power $P$. The condition $z^{*} \ll 1$ would also require drastic changes in the mean properties of the sources in a recent period, very short compared with the Hubble time, during which no other aspect of the Universe has altered substantially.

t These numbers can be found straightforwardly from differential counts, but it is easy to be misled by an integral plot such as Figure 3 into overestimating them. Note that any sudden discontinuity in the slope of differential counts tends to be manifested at a systematically lower flux (where $N$ is larger) in the integral plot, and so at first sight may seem more significant than it really is.

$+\dagger$ Even at high galactic latitudes, the main contribution to the radio background at $\sim 178 \mathrm{MHz}$ is believed to come from our Galaxy. The galactic spectral index is $\sim 0.4$ below $178 \mathrm{MHz}$ and steeper at higher frequencies. If the isotropic extragalactic contribution to the background has a different spectral index $\alpha$, it is possible to separate out the two contributions. If $\alpha$ equals the mean spectral index of known sources $(\sim 0.75)$ then the inferred extragalactic brightness temperature is $T_{408} \simeq$ $\simeq 2.8 \pm 0.1 \mathrm{~K}$ (Bridle, 1967). This temperature is inversely correlated with the assumed value of $\alpha$-for example if $\alpha=0.9$ it would fall by a factor $\sim 2$. For comparison, the integrated contribution from resolved sources down to the $5 \mathrm{C}$ limit corresponds to $T_{408} \simeq 1.4 \mathrm{~K}$. 
On the other hand, if $z^{*} \gtrsim 1$, redshift effects naturally lead to a sharp cut-off at low $S$. Alternatively the first sources might only have formed at the epoch corresponding to some redshift $z_{\max }$ which may not be substantially larger than $z^{*}$.

(ii) Even if the 'excess' sources occupied a narrow redshift range, the maximum of the $N / N_{0}$ curve would be broadened by any spread in $P$. Indeed, a luminosity function $\varrho(P)$ which had the same form at $z^{*}$ as it has locally would neither reproduce the sharp maximum in the curve nor be compatible with the observed limits on the integrated background. The evolution cannot therefore consist of a simple scaling of the local $\varrho(P)$ : instead, only the more powerful sources can be permitted to evolve.

The available freedom is reduced when, as has been customary, simple functional forms are adopted for the evolution (Oort, 1961 ; Davidson, 1962a, b; Longair, 1966; Doroshkevich et al., 1970). It is found that the curve can be fitted if the comoving density of powerful sources was $10^{2}-10^{3}$ times higher at redshifts $2-5$ than it is locally, but that the density cannot continue to rise sharply at even larger redshifts. However our most direct evidence on the Universe at redshifts $z \gtrsim 0.3$ (say), and on the very bright end of the radio luminosity function, comes (if their redshifts are cosmological) from optical observations of QSS. Without this information one could - with no less arbitrariness - explain the $N(S)$ curve by an evolution which peaked somewhere in the range $z \simeq(0.3-1)$. I shall therefore postpone further discussion of the form of the source evolution required in Friedman cosmologies until Section 4.

The interpretation of the counts in steady state theory was recently reviewed by Hoyle (1968). Since no secular change is permitted in this theory, one must invoke a fluctuation that gives rise to a local deficit of sources. If the QSO redshifts are not wholly cosmological, then the data would be consistent with a fluctuation extending out to $z \simeq 0.3$. The fluctuation could result from some cyclic variation in the source formation rate, with a time scale comparable with the Hubble time. If the variations were synchronous over the whole observed universe, no anisotropy need arise. However if the QSOs are cosmological, in which case the fluctuation must extend out to $z \simeq 2$, the steady state theory encounters greater problems.

An analysis of the Parkes $2700 \mathrm{MHz}$ counts by Wall (1970) shows that, despite the failure of these counts to reveal a slope significantly steeper than -1.5 , they still indicate the need for evolution. Even if the QSOs are local, the Parkes data alone are still irreconcilable with the strict steady state model. This is because even the radio galaxies have large enough redshifts to flatten the expected slope appreciably from the Euclidean value.

\section{B. IDENTIFICATIONS}

Optical counterparts have been identified for most of the stronger radio sources (and of course identifications are a prerequisite for determining the form of $\varrho(P)$, which is relevant to analyses of the counts), and the state of current work is illustrated in Figure 4. Though I shall not attempt a detailed review of identifications here, this diagram - due to Bolton (1969) - shows the key points in a succinct and illuminating way. One feature clear from the diagram is that the fraction of identified sources 


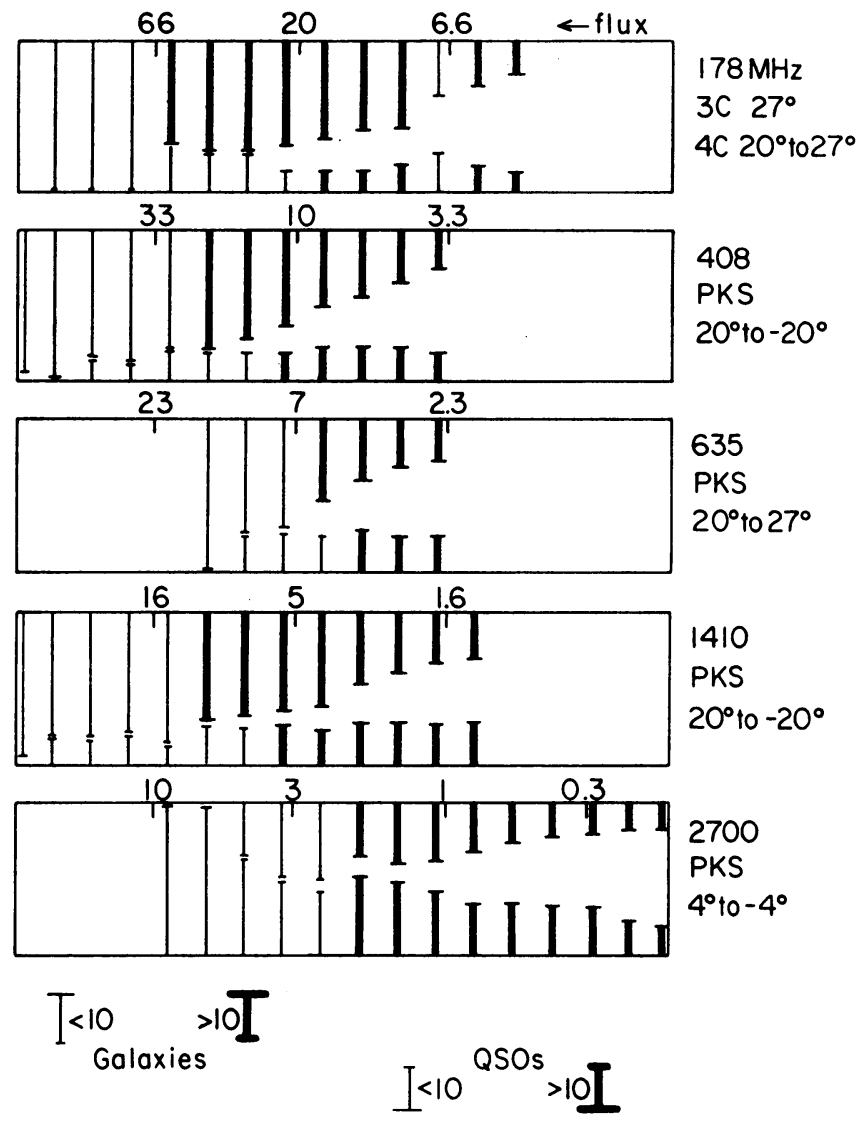

Fig. 4. The identification content of radio surveys at different frequencies. The abscissa is the flux density on a logarithmic scale, and the scales of the five diagrams are adjusted to give similar source densities at the same horizontal locations. The fraction of sources which are identified with radio galaxies above a given flux level is shown as a line extending downwards and the fraction which are QSS as a line extending upwards. The gap thus represents the unidentified fraction. The strongest sources in the $178 \mathrm{MHz}$ Cambridge surveys tend to be radio galaxies, whereas the opposite is true for the higher frequency Parkes surveys. The $N(S)$ curve for unidentified sources will obviously be steeper than the curve for all sources, because the unidentified fraction naturally increases as $S$ decreases. (reproduced from Bolton (1969).)

decreases towards lower flux. This reflects the existence of some correlation between apparent radio and optical brightness. It has the obvious consequence that the $N(S)$ relation for identified sources is somewhat flatter, and that for unidentified sources somewhat steeper, than the overall curve. A second important feature is that the ratio of QSS to radio galaxies among the identified sources is larger in the high frequency surveys. This is presumably related to the tendency for QSS to have compact selfabsorbed radio components with flat spectra. It is somewhat surprising that, for each survey, the ratio of QSS to radio galaxies varies so little with $S$.

The unidentified sources are believed to be mainly radio galaxies rather than QSS 
(insofar as there is a clear-cut division between these categories) for the following reasons. (i) The spectral indices of unidentified sources are, if anything, even steeper than those of identified radio galaxies; (ii) The fraction showing interplanetary scintillation (an indication that components of small angular size are present) is intermediate between the fractions for QSS and for radio galaxies. Thus, if the unidentified sources have linear dimensions characteristic of one of the two classes, but are more distant, they are likely to be galaxies; (iii) The distribution of optical magnitudes of the identified objects appears to cut off at the plate limit for galaxies but about a magnitude brighter for QSS. This indicates that many radio galaxies are, optically, too faint to be identified, whereas there is a genuine scarcity of apparently faint QSS.

It was first suggested by Véron (1966) that the steep $N(S)$ curve was attributable to the QSS. However, any statement of this kind depends on how one allocates the unidentified sources. If these are all grouped with the radio galaxies, the $N(S)$ slopes for the two categories are similar. But according to J. G. Bolton (private communication) recent spectral data suggest that $\sim 25 \%$ of unidentified sources are QSS. The initial slopes of the $408 \mathrm{MHz} \log N / \log S$ curve would then be $\sim-1.7$ for galaxies and $\sim-1.9$ for QSS.

There have been various attempts to plot separate $N(S)$ relations for types of sources picked out by radio criteria alone - for example, sources with high and low surface brightness (Fomalont, 1968; Lequeux, 1970). However, these are of only marginal significance, since any subdivision of the sources not only reduces the number in each category, but also introduces extra selection effects.

\section{OPTICAL COUNTS}

The key advantage of optical over radio observations is that they lead to a determination of the redshifts. However even without the redshifts one can perform optical counts of suspect QSOs as one does for radio sources, and it is interesting that these show a similar evolutionary trend. Searches of selected fields for objects with ultraviolet excesses led Sandage and Luyten $(1967,1969)$ to conclude that the number of QSOs increased by a factor $\sim 6$ for each unit increase in magnitude. They find $\sim 5$ QSOs per square degree with $B<19.4$, and estimate $\sim 100$ per square degree brighter than $B=21.4$. In a uniform Euclidean universe the predicted factor would be 4 rather than 6 , so this datum is equivalent to an anomalously steep $\log N / \log S$ slope. Braccesi and Formiggini (1969) obtained a sample of 300 objects chosen on the bases of both ultraviolet and infrared excess, a proven and efficient procedure for picking out candidate QSOs (Braccesi et al., 1968). They find that the $(2.5 \log N) / m$ relation for these objects has a slope of 1.74 , again indicating evolutionary effects.

Contamination of these samples by blue stars in our Galaxy would only enhance the significance of these results, since any reasonable distribution of stars would tend to flatten the slope below the Euclidean value. Nevertheless the statistical reliability of these counts does not approach that achieved by the radio observations. A further reason for caution may be that the selection criteria for choosing candidate QSOs, 
being based on observed colours, are inevitably not quite redshift-independent because the optical spectra are not straight power-laws.

\section{Information Added by QSO Redshifts (if Cosmological): The Luminosity-Volume Test}

If their redshifts are not due to the universal expansion, and their radiation did not set out towards us at a remote epoch, then QSOs plainly tell us nothing directly about cosmology. I shall return to consider the nature of QSO redshifts in Section 5, but for the moment let us suppose that QSOs are indeed vastly more remote than the furthest visible normal galaxies, and consider to what extent the redshifts augment what can be learnt from counts (radio and optical) alone.

In the absence of redshifts, the only procedure one can apply to a sample of QSOs is to plot the number-flux relationship. We have seen that, when this is done for a complete sample either at radio or at optical wavelengths, the number-magnitude gradient is too sharp to be compatible with any 'source-conserving' Friedman model with $\Lambda=0$. If the redshifts of the objects can be determined (and if - as we suppose throughout this section - these redshifts are entirely 'cosmological') one might imagine that a great deal of further information could be derived. However, it turns out, perhaps rather surprisingly, that knowledge of the redshifts adds very little. The main

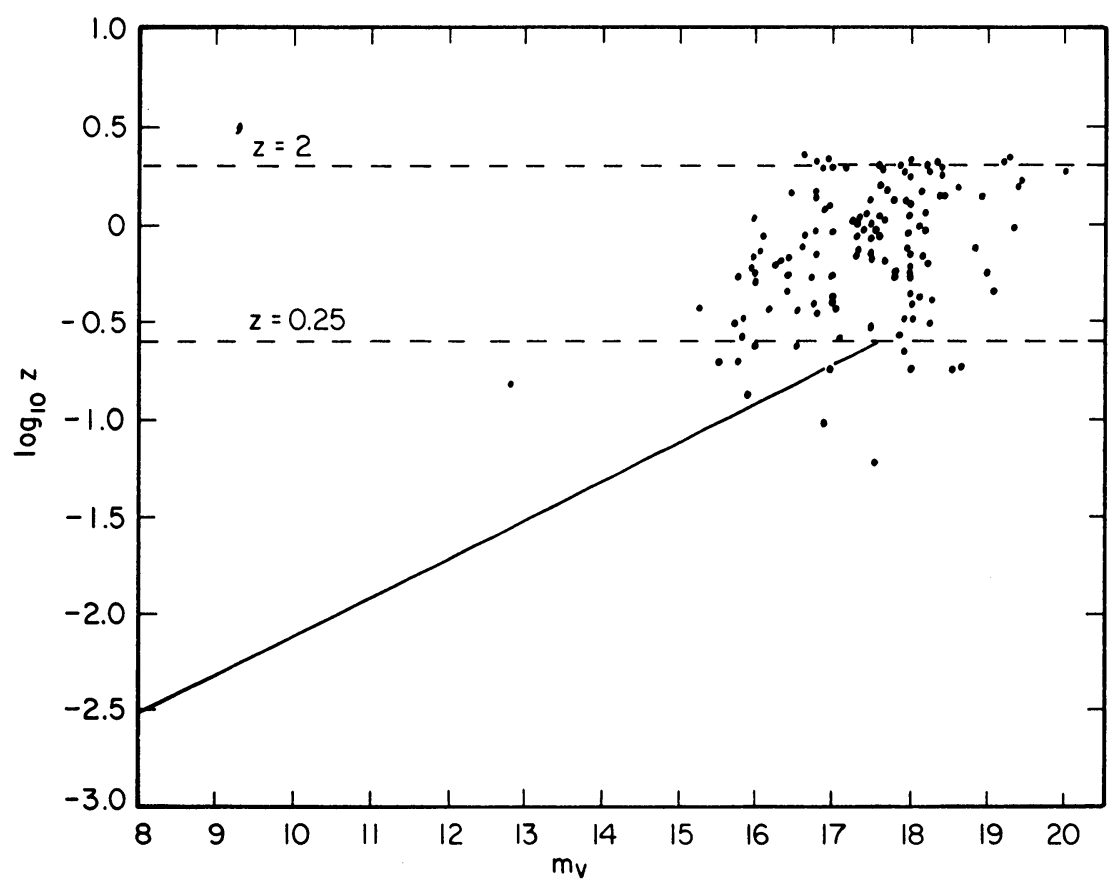

Fig. 5. The redshift-apparent magnitude relation for 136 QSOs. The full line is the Hubble relation for brightest cluster member galaxies (reproduced from Burbidge and Burbidge (1969)). 
reason is that there is hardly any correlation between the redshift and the magnitude. Figure 5 shows a plot of the $m-z$ relation for 136 QSOs. A similar 'scatter diagram' is obtained if radio flux is plotted against $z$ for QSS.

One would certainly not expect a well-defined relation between $m$ and $z$ such as exists for the brightest galaxies in clusters, if for no other reason than that the luminosity of some individual QSOs has varied by several magnitudes over the last few years. However, Longair and Scheuer (1967) were able to show, on the basis of the data available three years ago, that the lack of correlation between $m$ and $z$ cannot all be attributed to a broad luminosity function - there must be some $z$-dependence of the mean properties of the objects as well. This means that studies of QSOs with known redshift will be no more helpful than were radio source counts in discriminating between different cosmologies. Once again it will be possible to reconcile the data with any model by invoking suitable evolution, so the main interest of these investigations lies in the light they shed on the evolution and properties of the QSOs themselves.

Accepting, then, that the redshifts of QSOs indicate their distance, but that they do not constitute standard candles, what is the most efficient way to extract useful information from the data? The most popular technique - used first by Kafka (1967) and later, in various forms, by Rowan-Robinson (1968a, b, 1969); Schmidt (1968); and others - is the so called 'luminosity-volume test'. This test can be used even when the objects span a wide range of intrinsic luminosity, but to apply it rigorously one needs a sample complete down to some limiting apparent magnitude. Adopting a particular cosmological model, one then calculates, for each object, the volume $V$ (in comoving coordinates) out to its redshift $z$. One also calculates the maximum redshift $z_{m}$ which this same object would possess without appearing too faint to be included in the sample, and the corresponding volume $V_{m}$. (This stage in the calculation requires some information on the source's spectrum.) If the assumed cosmological model were the true one, and if the sources were indeed uniformly distributed, then $V / V_{m}$ should, on the average, be 0.5 . If it turned out to be systematically greater than 0.5 , implying that the sources tended to lie towards the edge of their accessible volume, this would indicate an evolutionary effect.

Longair and Scheuer (1970) have argued that, at least in the (actually unrealistic) case when the sample is limited by only one selection criterion, there is a close relation between luminosity-volume tests and source counts. The truth of this is clear in the following simple example. Supposing that we had redshifts for a complete set of objects in a certain field with $m<m_{\text {lim }}$ but knew nothing about their radio properties. Now $V / V_{m}$ is clearly very closely related to $m_{\text {lim }}-m$, but depends only slightly on the redshift. Thus $\left\langle V \mid V_{m}\right\rangle$ will depend mainly on the mean value of $m_{\text {lim }}-m$ - in other words, on how closely the magnitudes of the objects are concentrated towards $m_{\text {lim }}$ - and this information, of course, is contained in the counts. If there were no evolution the expected slope of the $(\log N) / m$ curve would depend on the mean redshift (since $z$-dependent quantities flatten it below the Euclidean slope), so an estimate of $\langle z\rangle$, as well as the slope, must be known before one can judge how strong the evolutionary effects are. However the relation between $m_{\text {lim }}-m$ and $V / V_{m}$ in Friedman models has 
only a slow $z$-dependence, so that a given set of values of $m_{\text {lim }}-m$ would lead to almost the same $\left\langle V \mid V_{m}\right\rangle$ if the individual redshifts of the objects were changed so as to leave $\langle z\rangle$ unaltered. Longair and Scheuer (1970) have shown this very convincingly by performing numerical experiments in which the redshifts of Schmidt's (1968) sample of 3CR QSS were randomly scrambled among the objects.

The luminosity-volume test does, however, yield information that is, in principle, genuinely independent of the counts in the more complex case when one has a sample limited by two selection criteria - for example a set of objects brighter than both a certain optical magnitude and a certain radio cutoff. The quantity $z_{m}$ (and therefore $V_{m}$ ) must now be defined as the redshift at which an object would disappear from the sample either because it became too faint optically or because it would be too faint radio-wise. With $V_{m}$ defined in this way, a uniform distribution of objects in such a sample would again yield $\left\langle V / V_{m}\right\rangle=0.5$. All published applications of the test refer to this more complex and more realistic situation (Rees and Schmidt, 1971).

Schmidt (1968) has applied this procedure to a sample of 33 identified QSS in the $3 \mathrm{CR}$ catalogue whose redshifts are known. These are believed to constitute a complete sample, over the area of sky covered, of QSS for which both $S_{178}>9$ f.u. and $m_{v} \lesssim 18.4$. (In fact the optical cut-off is defined more precisely in terms of the flux density at $2500 \AA$.) He finds that for standard Friedman models $\left\langle V / V_{m}\right\rangle \simeq 0.7$, almost independently of $q_{0}$; and for the steady-state model $\left\langle V / V_{m}\right\rangle=0.73$. This differs from 0.5 at a significance level of $0.01 \%$, thus providing strong evidence for cosmic evolution in these objects' properties. To estimate how strong the evolution had to be, Schmidt repeated the test in terms of a 'fictitious volume' $V$ ' calculated by weighting each volume element in proportion to a mathematically convenient increasing function of $z$. He found that, for a weighting factor with roughly the form $(1+z)^{6},\left\langle V^{\prime} \mid V_{m}^{\prime}\right\rangle$ was $\sim 0.5$. This indicates that the space distribution of the QSS in the sample is compatible with the assumption of a simple density evolution $\varrho \propto(1+z)^{6}$, though, as Schmidt stresses, no great weight should be attached to this particular functional form of the $z$-dependence.

Of the 33 objects in Schmidt's sample, 22 are 'radio limited' (in the sense that $z_{m}$ is the redshift at which $S_{178}$ would fall to 9 f.u.) and 11 are 'optically limited'. For the former, $\left\langle V / V_{m}\right\rangle=0.73$, and for the latter $\left\langle V / V_{m}\right\rangle=0.59$. This may indicate that the radio properties of the objects evolve more drastically than the optical properties, or else that the radio luminosity function is steeper than the optical one. It may seem that the optical data have added little, since the 'radio limited' objects carry most weight. In fact, however, the optical cutoff has selectively weeded out objects with high radio flux which, if $V_{m}$ were determined by the radio limit, would reduce $\left\langle V \mid V_{m}\right\rangle$ (though as Longair and Scheuer point out, the specific association of particular redshifts with specific objects does not affect the result).

The main virtue of the luminosity-volume test is that it is unaffected by the tendency for a decreasing proportion of sources to be identified at lower radio flux density (which happens when there is any positive correlation between the radio and optical luminosities of the objects concerned). Actually the radio evolution of 3CR QSS is 
so strong that it is apparent, despite this effect, from the radio counts alone, but this contingent fact does not mean that the luminosity volume test in principle adds little to the counts - it is easy to devise hypothetical samples of objects whose distribution of radio and optical brightness is such that the luminosity-volume test would reveal evidence for evolution which would not emerge from either optical or radio counts.

The luminosity-volume technique provides a convenient method for determining the luminosity function. Schmidt in fact did this for his 3CR QSS but the numbers involved were small, and the analysis was based on the (probably unwarranted) assumption that the radio and optical luminosity functions were uncorrelated.

In an attempt to derive the space distribution and luminosity function of QSOs as a whole, Schmidt (1970) has investigated a sample consisting of 20 objects from a Sandage-Luyten field, which is believed complete in the optical magnitude range 17.5-18.5. As already mentioned, counts of objects in the Sandage-Luyten fields show that the number per square degree rises by a factor $\sim 6$ per unit magnitude increment. This steep number-magnitude relation can be reproduced, in the standard Friedman models, by an evolution $\propto(1+z)^{6}$. By making this assumption for the objects in his sample, Schmidt is able to derive a luminosity function - it turns out that the coordinate density of QSOs increases by a factor 3 or 4 per magnitude, with probably an even steeper cut-off at the bright end. Schmidt can then (again assuming the $(1+z)^{6}$ evolution law) predict the expected number and redshift distribution of QSOs of all magnitudes. This leads to the conclusion that $24 \%$ of 19 th magnitude QSOs should have $z$ in the range 2.5-3. Out of 20 such objects observed, none has such a large redshift, and only one 18th magnitude QSS has yet been found with a redshift in this range (Lynds and Wills, 1970). This means that it is not consistent to apply the $(1+z)^{6}$ evolution law out to $z \simeq 3$. However, one obtains adequate agreement with the data by stopping the evolution at $z \simeq 2.5$ and supposing that at earlier epochs the coordinate density of QSOs stayed constant (and equal to its value at $z \simeq 2.5$ ).

A further very important point emerges when this sample of all QSOs is compared with Schmidt's earlier (1968) sample of QSS in the 3CR catalogue: it is found that the redshift distribution is the same for the $3 \mathrm{CR}$ objects around 18th magnitude as for the objects in the non-radio sample. This is highly significant, for the following reason. If the radio and optical luminosities were independent, the 3CR objects should tend to have smaller redshifts than the non-radio QSOs, because at large $z$ only those QSOs which were exceptionally bright radio-wise would appear in the 3CR catalogue, whereas at small $z$ even quite weak radio sources would be included. Schmidt interprets this result as an indication that the radio and optical luminosities are not independent, and tentatively suggests that the radio luminosity function may scale with optical luminosity. This would mean that the ratio of the apparent brightness of QSOs at radio and optical wavelengths - i.e. $m_{\mathrm{rad}}-m_{v}$, which can be thought of as a generalized 'colour' - was distributed according to a universal function independent of optical luminosity and (apart from a dependence on the difference between optical and radio spectral indices) of redshift. 


\section{The Inferred Evolution, and Possible Alternatives to it}

The necessity for evolutionary effects in the mean properties of the stronger sources is apparent from radio data alone. However without knowing the distances of QSS one cannot decide whether the major 'excess' of sources lies at a redshift as great as, say, $\sim 5$, or alternatively only at $z \simeq 0.3$ (the latter being large enough to satisfy the Olbers-type constraints discussed in Section 2, as well as being comparable with the largest redshifts observed for 'bona fide' galaxies that indubitably obey Hubble's law). If QSS redshifts are 'cosmological' we have seen that the luminosity-volume test reiterates the message of the source counts. Also, more importantly, the redshift data pin down the epoch of the main excess - both for QSS and for radio-quiet QSOs - in the range $z \simeq 2-3$.

The most dramatic feature of this evolution is its steepness; the observations imply that in Friedman models, irrespective of $q_{0}$, the density of powerful sources must increase by a factor $10^{2}-10^{3}$ per comoving volume (i.e. over and above the $(1+z)^{3}$ expansion factor). Since no astrophysical considerations place any constraints on this evolutionary behaviour - as they do, for example, on the $z$-dependence of the optical luminosity of normal galaxies - the observations provide no help in determining the geometry of the Universe. It is therefore more interesting to invert the problem, and try to learn something about the epoch-dependence of the source parameters.

Forms of radio evolution have generally been considered in which either the luminosity or the comoving density of a class of sources varies as some function $f(z)^{*}$. Much attention has been given to the case $f(z) \propto(1+z)^{n}$. Longair (1966) for example, was able to fit the data on the supposition that $\varrho(P) \propto(1+z)^{5.5}$ for all sources with $P_{408} \gtrsim 5 \times 10^{25} \mathrm{~W} \mathrm{~Hz}^{-1}$ ster $^{-1}$. These steep power laws must be truncated at some redshift $z_{\max }$ to ensure convergence. Longair originally chose $z_{\max }$ to be 3 or 4 , and tentatively associated this value with the epoch of galaxy formation. The subsequent optical studies by Schmidt and others suggest that $z_{\max } \simeq 2.5$ might be a better estimate. Of course there are no clear astrophysical grounds for expecting $f(z)$ actually to resemble a power law (though I shall return to this point in a moment), and this choice was made principally for computational convenience. Another mathematically tractable choice is $f(z) \propto e^{-k t}$ where $t$ is cosmic time. This corresponds to $\exp \left(\frac{2}{3} k t_{0}\left(1-(1+z)^{-3 / 2}\right)\right)$ in an Einstein-de Sitter $\left(q_{0}=\frac{1}{2}\right)$ model, and to $\exp \left(k t_{0}\left(1-(1+z)^{-1}\right)\right)$ in a very low density universe, where $t_{0}$ is the 'Hubble time'. It is unnecessary in this case to impose an arbitrary truncation $z_{\max }$ to ensure that the counts converge. Exponential evolution has been considered by Rowan-Robinson (1968a) and by Doroshkevich et al. (1970).

Since there is no reason for the evolution to obey any simple analytic law, it may be

* One should note that the often-drawn distinction between 'density evolution' and 'luminosity evolution' has little significance unless one can correlate each class of radio source with a particular type of optical counterpart. We do not yet know enough about the connection between optical and radio properties of QSS and radio galaxies to be able to do this. All that can be meaningfully discussed on the basis of the radio evidence is the $z$-dependence of the luminosity function, 'density evolution' being merely a special case. 
premature to discuss the form of $f(z)$ until we know whether all sources, or only some particular types, partake in the evolution. It is nonetheless amusing to speculate on some physical effects which could contribute to the epoch-dependence of radio source properties. A feature common to almost all proposed models is that the active lifetime of a strong radio source is probably no more that $10^{6}-10^{7} \mathrm{yr} *$. This is much shorter than the Hubble time at all relevant redshifts. At any cosmic epoch, we must therefore be observing sources on every part of their evolutionary track, the relative number of sources (per unit volume) at each particular stage of development being inversely related to the rate at which they evolve through that stage. Consequently observations of QSOs at large redshifts do not reveal objects which are individually younger - in the sense of having experienced a shorter lifetime - than similar nearby objects. The inferred epoch-dependence of $\varrho(P)$ must therefore reflect a change either in the evolutionary track followed by each source, or in the source birth-rate (or more probably in both these things).

It is natural to suppose that compact sources eventually develop into extended doubles, and to consider whether all radio sources can be explained as different stages in a single evolutionary track. That this might indeed be the case was suggested by the work of Aizu et al. (1964), who plotted volume emissivity against $P$, and by Heeschen (1966) who plotted surface brightness against $P$. Ryle and Longair (1967) have proposed a possible general scheme for interpreting the strong radio source data. They assume that the required energy of $10^{60}-10^{62}$ erg is generated in a single event, and that the resulting plasma clouds travel out from the parent object at speeds of $0.1-0.9 c$, eventually fading as they expand adiabatically into the intergalactic medium. During the early stages, the properties of the source will depend on the details of the initial 'violent event'. However the behaviour during the later extended phase may be insensitive to the properties of the primary outburst. During this phase, the expansion rate (and thus the lifetime) would be determined by the 'ram-pressure' of the external medium, and would therefore be related to the density $\varrho_{g}$ of the intergalactic gas (De Young and Axford, 1967; Rees and Setti, 1968; Christiansen, 1969; Mills and Sturrock, 1970). The epoch-dependence of $\varrho_{g}$ depends on how uniformly the gas is distributed, and on whether it is concentrated in clusters, but we might guess that it would vary as $(1+z)^{3}$ even though its present value is highly uncertain. The expansion rate of sources into the intergalactic medium depends - other things being equal - on $\varrho_{g}^{-1 / 2}$. Thus, even if neither the source formation rate nor the character of the initial explosion depended on $z$, this effect alone could cause the coordinate density of powerful sources to vary as $(1+z)^{3 / 2}$. The quantity $\rho_{g}$ is the most important relevant parameter whose $z$-dependence can be quantified, and it is interesting that it could contribute a power-law dependence to $f(z)$. But this is probably only a subsidiary effect, since the main factor governing $f(z)$ is likely to be the source birth-rate, or the probability of occurrence of 'violent events'. (Schmidt's evidence that radio-quiet

* The same objects could however continue to function at a lower power level for a much longer period. Schmidt's (1966) estimate of the lifetime of sources associated with elliptical galaxies suggests that this does indeed happen. 
objects display the same evolution as QSS (Section 3) supports this view). This is not the place for a review of current ideas on the nature of 'violent events' (but see Woltjer, 1971). According to the 'spinar' or stellar collision models, the evolution time-scale before catastrophic collapse ensues depends on various powers of the initial density, the mass, and (for the 'spinar' model) the magnetic field. Thus, if all galactic nuclei formed at a certain redshift with a uniform spread in these parameters, the event rate would vary as a negative power of time; objects forming closest to the verge of instability would collapse first, and the event rate would decrease monotonically. To mention one further possibility, if 'violent events' are an accretion phenomenon (Sturrock and Feldman, 1968) the luminosity would vary as $\varrho_{g}$, and therefore perhaps as $(1+z)^{3}$.

It is at least suggestive that all the above effects indicate a power-law form for $f(z)$. These arguments also suggest that galaxy formation occurred around $z \simeq 2-3$, which is consistent with many current theories (see Field, 1970 for a review of this subject).

Refinements of the luminosity-volume test should, when more redshifts are available, be able to determine whether the evolution of QSOs obeys a power law or an exponential function. (The latter would tend to have a steeper $z$-dependence at small redshifts.) It should also be possible to determine the evolution separately for different luminosity classes, and hence to discover how the whole luminosity function evolves.

There seems no reason to regard this drastic epoch-dependence of source properties as unacceptable, especially if one recalls that the epochs concerned take us $\sim 80 \%$ of the way back in time to the 'big bang'. But it is interesting to consider two less conventional ideas which would at least reduce (even if they do not entirely eliminate) the amount of evolution that the observations imply. These respectively involve (a) Lemaître cosmological models; and (b) gravitational lenses.

\section{A. LEMAÎTRE MODELS}

The surprisingly large proportion of QSO redshifts around $z \simeq 2$ led Kardashev (1967), Shklovsky (1967) and Petrosian et al. (1967) to revive the cosmological models associated with the name of Lemaître. When $\Lambda$ is non-zero, there will always be a particular stage in the expansion of a model universe, corresponding to some redshift $z_{c}$, where the cosmical repulsion exactly counterbalances gravity. At this stage in the expansion the deceleration would be zero. If the expansion rate or density is so adjusted that the expansion almost stops at $z_{c}$ then the universe may undergo a long, almost static, 'coasting phase'. In such a universe one might expect to observe a large number of objects with redshifts $\sim z_{c}$ for two reasons. First, at $z_{c}$ a large increase in coordinate distance, and in coordinate volume, corresponds to only a small increment in $z$; and, second, the fact that in these closed universes there is time for light to make one or more complete circuits during the 'coasting phase' means that there are 'poles' *,

* If $\varepsilon$ (defined overleaf) is $\ll 1$, there may be a large number of poles with $z \simeq z_{c}$. The most remote pole (in terms of coordinate distance) can be at an arbitrarily large redshift which is sensitive to the precise length of the coasting phase. As $\varepsilon \rightarrow 0$, and the model tends to the standard Eddington-Lemaitre model, the redshift of the nearest pole is given by $\left(z_{c}-z\right) \simeq 0.04\left(1+z_{c}\right)$. 
and any object lying close to one of these is magnified and therefore more likely to be detectable.

Kardashev and Shklovsky were concerned to explain an alleged (Burbidge, 1967) peak in the QSO redshift distribution at $z=1.95$. This apparent peak was first found in the distribution of QSO absorption redshifts, and could be attributed, in a Lemaître model, to the high probability of there being an object with $z \simeq z_{c}$ lying along the line of sight. However the peak was later found even more sharply $(z=1.955)$ in the QSO emission redshift distribution. A Lemaître model with an extremely long coasting phase was required, in which the parameters are adjusted to within a factor $\varepsilon \simeq 10^{-4}$. Petrosian et al. dealt only with the general pile-up of redshifts around $z \simeq 2$, and so needed a less finely adjusted - and so perhaps, a priori, less improbable - version of the model with $\varepsilon \simeq 0.02$. (The duration of the coasting phase is proportional to $-\log \varepsilon$ ).

Such models account for the large number of QSO redshifts around 2, and also the absence of observed objects with $z \gtrsim 3$, without requiring any very strong epoch dependence of the QSO coordinate density. However one cannot dispense with evolution altogether in these models (at least unless one is prepared to consider models with $z_{c}<1$, which lead to other problems). This point is discussed by Longair and Scheuer (1970). The need for evolution is inferred from: (a) application of the luminosityvolume test to objects with redshifts small enough to be unaffected by the peculiar features of Lemaître models; and (b) the source counts. As regards (b), it is true that a $\log N / \log S$ slope steeper than the Euclidean value is in principle obtainable in Lemaître models (McVittie and Stabell, 1967). However this purely geometrical steepening only occurs if sources with $z \simeq z_{c}$ make the main contribution to the counts. It is therefore unacceptable as an explanation of the observed $N(S)$, since the identifications of $3 \mathrm{C}$ sources are complete enough to show that this cannot be the case.

The property that light can perform a complete circuit of the universe allows a further interesting test of Lemaitre-type cosmologies, since the same source may be detectable at two antipodal points. Solheim (1968a, b) claimed that such an effect existed, but its significance was questioned by Piper (1968), Petrosian and Ekers (1969) and Audretsch and Dehnen (1969). However, it is in any case unclear whether one should expect a marked effect of this kind in realistic Lemaitre models, for several reasons: (i) The radiation reaching us via the two routes would have been emitted at different times, and if this time difference $t_{D}$ exceeds the active lifetime of a strong source (perhaps $\sim 10^{6} \mathrm{yr}$ ) no antipodal coincidences would be observed; (ii) If they have substantial peculiar velocities, sources near the "poles" may traverse a significant angular distance during $t_{D}$ and so even if a source lasted for a time longer than $t_{D}$ its two images would not appear antipodal; (iii) If clusters of galaxies, or similar large-scale inhomogeneities, had already separated out by the end of the coasting phase, then the radiation from a QSO or compact source near a pole would be split into several 'ghost images' (Petrosian and Salpeter, 1968).

\section{B. GRAVITATIONAL LENSES}

In a series of papers, Barnothy and Barnothy (1968 and references cited therein) have 
suggested that QSOs are not in fact superluminous objects, but are instead merely compact sources (such as Seyfert nuclei) which have been focussed and magnified by a massive object along the line of sight acting as a gravitational lens. This proposal has the obvious attraction that it removes the specially severe energetic requirements associated with QSOs. But it also has the great virtue that it naturally accounts for the apparent increase with redshift in the QSO coordinate density - since the number of potential lenses along the line of sight, and the magnifying power of each, increases with $z$. The criticism most often levelled against this theory is that the effect requires such a precise alignment of the lens and the source, and is therefore so improbable, that one cannot account for all QSOs in this way. Quantitative estimates have been made by the Barnothys and by Pacholczyk and Weymann (1968). The latter authors estimated that if the lenses each had a mass $M_{L} \simeq 10^{10} M_{\odot}$ and if their number was such as to provide a smoothed out density $\varrho_{L} \simeq 7 \times 10^{-31} \mathrm{~g} \mathrm{~cm}^{-3}$, then the ratio of QSOs to Seyferts fell short by $\sim 100$ of the observational value. However this ratio scales as $\varrho_{L}$, and the maximum magnification depends on $M_{L}$ so that if a density of $10^{-29} \mathrm{~g} \mathrm{~cm}^{-3}$ were present in the form of objects with masses $\sim 10^{11} M_{\odot}$, the correct number of QSOs could conceivably be explained. (Pacholczyk and Weymann considered a Euclidean universe, and the calculated probability would obviously increase if the higher density of the universe at large $z$ were allowed for). In view of the willingness of many theorists to invoke hypothetical entities such as 'dead galaxies' or black holes in other contexts, it would seem unfair to discard the gravitational lens model on probability grounds.

Perhaps the one crucial test of the gravitational lens theory would be to determine whether the apparent source evolution can be entirely attributed to objects with angular size $\lesssim 1^{\prime \prime}$, since if the lenses do not exceed a galactic mass they cannot give rise to magnified images larger than this.

\section{The Nature of QSO Redshifts}

In the preceding sections the redshifts of QSOs have been assumed, wherever they are relevant, to be 'cosmological'. However, the question of the actual nature of these redshifts is still to some extent an open one, and in view of its importance for cosmology (as well as its obvious bearing on the astrophysical nature of QSOs) it may be useful to summarise some of the relevant arguments. If the redshifts are not cosmological - or if they have a substantial non-cosmological component - they could be either: (a) gravitational implying that the spectral line emission originates in a gravitational potential well so deep that relativistic effects are important; (b) local Doppler, meaning that QSOs possess relativistic peculiar velocities; or (c) 'metaphysical'. This last category is intended to include interpretations involving fundamentally new laws of physics, or as yet unenvisioned aspects of space-time which may manifest themselves on a cosmic scale. Although it is worth trying to assess the relative unlikelihood of (a), (b), and (c), the more basic question for cosmology is simply whether QSOs are 'local' or whether their redshifts obey some form of 
Hubble law. The evidence of this question may be discussed under four headings.

\section{(i) Direct Indications of Location Relative to Other Objects}

The most straightforward way to determine a QSO's distance is to find another object, plainly physically associated with it, whose distance is less ambiguous. The low redshift $(z=0.09)$ object B264, which had previously been listed as a QSO, was found by Bahcall et al. (1969) to lie in a cluster of galaxies with the same redshift. There can therefore be no doubt that its redshift is essentially cosmological. The same authors also point out that 5 of the 6 known QSOs with $z<0.2$ lie within the boundaries of clusters of galaxies, whereas such clusters cover less than $10 \%$ of the sky. Since these clusters would have redshifts in the range $0.1-0.2$, this argues in favour of a genuine physical association in the other cases also. It is, however, unfortunate that the one QSO in this redshift range that does not appear to be associated with a prominent cluster is the archetype quasar 3C 273. The other objects are intrinsically faint (B264 being actually $\sim 1$ magnitude fainter than a typical brightest cluster member at the same redshift) and maybe should be classed as $\mathrm{N}$-galaxies. Nevertheless, as there may be a continuous gradation from one type of emission-line object to the other, it is fair to take these associations with clusters as support for the cosmological interpretation of all emission line redshifts.

Plates of 3C 273 and 3C 48 by Sandage and Miller (1966) taken with special emulsions failed to reveal any clustering in the vicinity of either object. This does not preclude these QSS belonging to small groups. (At the distance of 3C 273 the only members of our Local Group that would appear on Sky Survey plates are the Galaxy and M31; and only 2 of the 15 other known members would be near the limit of detection (Bahcall and Bahcall, 1970).) Of course, there would be no hope of seeing any ordinary galaxy at the distances corresponding to the redshifts of most of the QSOs in Figure 5. However, the detection of absorption features in a QSO's spectrum attributable to a distant observed cluster could set a useful lower limit to the distance (Bahcall, 1969).

It may be trite and superfluous to point out that whereas the association of QSOs with clusters at the same redshift would be strong support for the 'cosmological' viewpoint, the absence of such associations is by no means equally firm evidence for a contrary conclusion.

Some remarkably suggestive cases when a QSO (or other compact object) appears to be associated with a galaxy with a different redshift should also at least be mentioned under this heading. For example, Markarian 205 - an $N$-galaxy - lies within a spiral arm of a nearby galaxy (Weedman, 1970). There are also cases - for example NGC 772 and its companions (Arp, 1970a) - where a physical bridge appears to link a compact object with a large galaxy of much smaller redshift. It is hard to form any reliable estimate of the probability that these strange coincidences should result from chance superpositions, but the present data at least seem sufficiently convincing to warrant further systematic investigation. If further examples are searched for according to some preassigned criteria, it should eventually be feasible to test the significance of any associations that may be discovered. 
(ii) Constraints on 'Local' Theories from Counts, Integrated Background, Isotropy, etc.

Any interpretation which reduces the estimated distances of the observed QSOs is likely to involve an increase in the integrated contribution from distant unresolved objects. The known limits on the extragalactic radio background therefore constrain such interpretations.

If QSOs have gravitational redshifts, and resemble the model proposed by Hoyle and Fowler (1967), then their individual masses should be of galactic order; the objects would have lifetimes $\sim 10^{10}$ yr and could perhaps be distributed through the universe in the same manner as galaxies. Considerations of the integrated radio background then require that the observed radio QSS must be at least $\sim 50 \mathrm{Mpc}$ distant. If the distances were indeed of this order, then QSO counts would have a Euclidean slope. One would then be forced to seek some explanation of the steep $N(S)$ curve that did not involve QSOs (for example, in terms of cosmological evolution of radio galaxies alone). However neither the optical counts, nor the radio data (RowanRobinson, 1969), are compatible with a Euclidean distribution of QSOs: indeed it seems more likely that QSS are largely responsible for the steepness of the total $N(S)$ curve. One must therefore invoke a local 'hole', with scale $\gtrsim 50 \mathrm{Mpc}$, to account for the deficiency in the relative number of bright sources. Pooley and Ryle (1968) emphasise that the isotropy of the $4 \mathrm{C}$ survey (established to a precision of at least $5 \%$ ) means that we must lie within $\sim 1 \%$ of the centre of any such hole - and the probability that a random point in a sphere should be thus located is 1 in $10^{6}$. However, a local deficit attributable to a 'time hole', such as might occur in cosmologies involving continuous creation (Hoyle, 1968) automatically leads to complete isotropy; if the QSO redshifts have a cosmological component of 0.1-0.3, and the 'hole' extends out to $z \simeq 0.3$, this kind of generalised 'steady state' model can satisfy all observational constraints.

Note also that these Olbers-type considerations provide no firm arguments against the local Doppler theory (Terrell, 1966, 1968), even though the distances of the QSOs are there envisaged as only 1-10 Mpc, because the sources responsible for the steep counts would then lie in a thin shell around us, rather than constituting the inner edge of a population extending out to the Hubble radius. High isotropy would be ensured if the QSOs were ejected from the centre of our own Galaxy. The integrated background would limit the frequency of similar occurrences in other galaxies.

\section{(iii) Redshift Periodicities}

After his earlier suggestion that the redshift $z=1.95$ had special significance, Burbidge (1968) claimed that a histogram of redshifts of emission line objects revealed a significant tendency for $z$ to cluster around multiples of 0.061 , the peaks around 0.061 and 0.61 being specially marked. A similar claim was earlier made by Wilson (1967). A power spectrum analysis of 186 redshifts by Plagemann et al. (1969) revealed periodicities that were at best marginally significant at the $5 \%$ level, contrary to the 
earlier conclusions of Cowan (1968). Of course, if the 'spikes' in the redshift histogram were really exceedingly sharp, they might still be highly significant even though nothing clear-cut emerges from the harmonic analyses. However a plot of redshifts 'modulo 0.061' shows that the peaks are not in fact very sharp (P. Martin, private communication). Therefore, any effect that was statistically significant should have been found by Plagemann et al.

If any effect of this kind were established beyond doubt, it would force us towards a 'metaphysical' interpretation of QSO redshifts. (Though a single well-defined preferred redshift, rather than a periodicity, could occur in a Lemaître type model.)

\section{(iv) The Relative Difficulty of Devising Plausible QSO Models on the Basis of the Rival Hypotheses}

When clear-cut evidence is lacking, our views on the nature of QSO redshifts are naturally influenced by the relative success of attempts to construct plausible models based on the various alternative possibilities. Plainly no great weight should be attached to these subjective assessments - they may reveal more about the limits of conventional physics, or the limited ingenuity of astrophysicists, than about the QSOS themselves - but a few brief points may be worth a mention.

It was principally the massive energy requirements estimated for cosmologically distant QSOs that led many workers to investigate alternative interpretations for the redshift. Comparable energies were already known to be involved in extended radio sources like Cygnus A, but recent developments in infrared astronomy have shown that the energy output of Seyfert nuclei over their estimated lifetime of $10^{8} \mathrm{yr}$ is no less exorbitant. Most astrophysicists who have considered the origin of this energy have regarded the gravitational binding energy of a massive object as the most promising candidate. Recently Lynden-Bell (1969) and Bardeen (1970) have shown explicitly how up to $42 \%$ of the rest energy of infalling material could be transformed into electromagnetic radiation. If QSOs were indeed powered by the type of mechanism envisaged by these authors, the energetics, even on the cosmological hypothesis, could be comfortably satisfied by a mass of $\sim 10^{9} M_{\odot}$. The rapid variability of QSS implies small linear dimensions, and therefore (especially on the cosmological interpretation) high radiation densities within the emitting region. The problem of interpreting the non-thermal radiation without an 'inverse Compton catastrophe' was first pointed out by Hoyle et al. (1966). However, as a result of recent very-long-baseline radio interferometry, the strictest upper limits on the dimensions of the emitting region are now sometimes set by an angular size criterion; the estimated radiation density within the object is then independent of what distance is assigned to it. Several authors subsequently devised synchrotron-emitting models for QSOs which overcame the objection emphasised by Hoyle et al. Also, the discovery of pulsars led to the realisation that coherent emission mechanisms (to which this objection would not in any case be relevant) may play a role in QSOs also.

For the above reasons and others (see, e.g., Schmidt (1969) for references to further work) the theoretical case against cosmologically distant QSOs seems less compelling 
than it once did. At the same time, the difficulties with the gravitational and local Doppler interpretations of the redshifts have become more apparent.

It now seems clear that the central gravitational redshift in the Hoyle-Fowler model cannot be as large as $\sim 2$ without the system becoming subject to general relativistic instabilities (though rotating systems, which have not yet been analysed in detail, could turn out to be more stable). Also, some important features of the optical spectra of QSOs are hard to incorporate into the Hoyle-Fowler model. The presence of forbidden emission lines, especially [OII] $\lambda 3727$, implies an upper limit to the density of the thermal gas. The volume available in the potential well is proportional to $M^{3}$ ( $M$ being the total mass), and the thermal luminosity has the same dependence when the density has the maximum allowed value. The distance of a typical observed object would then be $\propto M^{3 / 2}$. Since the objects must not collectively contribute more than $\sim 10^{-29} \mathrm{~g} \mathrm{~cm}^{-3}$ to the mean density of the universe, one obtains a lower limit to $M\left(\sim 10^{13} M_{\odot}\right)$, such that the distance of observed QSOs must be at least $\sim 200 \mathrm{Mpc}$. The absorption lines must, in the model, be due to discrete clouds with smaller gravitational redshifts situated on the steep part of the potential well. The narrowness of the observed lines then implies that such a cloud must be $\lesssim 10^{-3}$ times the size of the whole system. This implies that the continuum being absorbed must originate from a concentrated source at the centre, and if this were a massive object the potential well would no longer have the parabolic form needed to ensure that the emission lines have a well defined redshift. Also the inferred low density and moderate excitation of the absorbing material are hard to reconcile with a location so close to the intense central continuum source.

The evidence from the work of Sandage and Luyten (1969) that there may be $\gtrsim 10^{7}$ detectable QSOs aggravates the energy requirements of the 'local Doppler' model; even if the mass of each QSO were only $10 M_{\odot}$ the energy released in the hypothetical explosion at the Galactic Centre would be at least comparable with that of a cosmological QSO. Moreover, the explosion has to be so contrived that it ejects many discrete objects rather than a few large clouds of debris. If we recall also that both the integrated background limit and the absence of blueshifts require that such explosions are very improbable (so that it is a priori unlikely that our Galaxy exploded sufficiently recently for the ejected QSOs to still be visible), one must surely conclude that this form of 'local' interpretation is less credible than either the 'gravitational' or the 'metaphysical' category.

There is, sadly, still no observation which clinches this key question, but the above remarks will have indicated some that may do so in the future. The cosmological interpretation leads to no inconsistencies, and is the most 'conservative' in that it entails merely an increase in the scale on which known astrophysical processes operate, rather than forcing us to invoke some qualitatively new phenomenon which operates in QSOs and nowhere else. The one reason for feeling at least slight unease about the 'cosmological' view is that, if one adopts this as a hypothesis, the data lead to the conclusion that the properties of QSOs must evolve with time in such a way that they mask the correlations which one would expect to find (at least in standard homoge- 
neous cosmological models based on general relativity) if $z$ indeed was a measure of distance. In fact Longair and Scheuer (1967) showed that the only correlation between QSO magnitudes and redshifts was precisely what would be expected on the gravitational hypothesis. The only significant evidence so far obtained that $z$ is a distanceindicator is Miley's (1968) correlation between fringe visibility and redshift for steep spectra QSS.

\section{Some Other Potential Cosmological Tests}

\section{A. RADIO TESTS}

The redshift-dependence of angular diameters gives us, in principle, another way to discriminate between different cosmological models. Radio sources have, in this connection, the advantage of being frequently large enough to be resolved even at cosmological distances. However their great range of linear dimensions means that they do not serve as 'standard rigid rods' (any more than as 'standard candles'), so that one can, at best, hope only to discern some statistical trend in a relation involving much scatter.

Miley (1968) and Bash (1968) have correlated fringe-visibility with redshift. Flat spectrum objects would be expected to have self-absorbed components which would be unresolved even at small distances, but Miley found a significant correlation for QSS with steep spectra. This is important evidence that redshift and distance are indeed correlated, but (accepting this) one cannot safely infer anything further because, owing to observational selection, the only large redshift objects studied would be those with high $P$, which may differ systematically from the typical low redshift source in the sample. Legg (1970) plotted the angular sizes of 57 double radio sources against redshift. On the assumption that there is a well-defined (and redshift-independent) upper limit to the linear size of strong sources, he found that the data agreed better with the predictions of a de Sitter (steady-state-type) model than with Friedman models. However there may be evolutionary effects in the typical sizes of sources - for example the components may separate more slowly at large redshifts because they are moving through an external medium of higher density - and, in our present state of ignorance these effects are just as unpredictable as the evolution in the luminosity function. Thus, as Legg points out, even if we could be sure that there were no selection effects in the sample, this test cannot be used to distinguish between different Friedman models.

Longair and Pooley (1969) have carried out a different kind of angular diameter test which does not involve knowledge of the redshifts. Using the data on the size distribution of $3 \mathrm{C}$ sources, they calculated that, neglecting evolutionary effects, there should only be $\sim 2$ faint sources in the $5 \mathrm{C}$ surveys with angular sizes $\gtrsim 70^{\prime \prime}$. The fact that $\sim 10$ such sources are found therefore indicates evolution of large diameter radio sources with $P_{178}$ in the range $\left(10^{24}-10^{26}\right) \mathrm{W} \mathrm{Hz}^{-1}$ ster $^{-1}$. This evolution is less drastic than that implied by the $N(S)$ relation, but the latter refers only to more powerful sources. 


\section{B. TESTS USING QSOS}

If their redshifts are indeed a measure of distance, then the QSOs furnish us with a probe for studying the universe at early epochs, back to a time when - in standard Friedman models - the Universe was only $20-25 \%$ of its present age. Studies of the distribution over the sky of QSOs in specific redshift ranges could, in principle, test the homogeneity of the universe on scales larger than superclusters. Strittmatter et al. (1966) claimed that QSOs with $z \gtrsim 1.5$ were not in fact uniformly distributed over the part of the sky accessible to observation, but that there was one concentration towards the North Galactic Pole and another in the South Galactic Hemisphere. If this effect were genuine, it could be attributable to clustering of QSOs on a scale of $\sim \frac{1}{2}$ the Hubble radius. Although the number of known redshifts has roughly trebled since this work was done, the status of Strittmatter et al.'s assertion remains unclear. (Arp (1970b) has claimed that QSOs are not randomly distributed over the sky, but tend to surround nearby galaxies. If significant, this would argue in favour of noncosmological redshifts.) The main difficulty is that many QSOs are detected in the course of intensive surveys of small areas of sky, and it is consequently hard to evaluate the many selection effects in a quantitative way. Studies of the sources in the Cambridge surveys (Holden, 1966; Hughes and Longair, 1967) have revealed no evidence for anisotropies on any angular scale. However, the QSOs in principle provide an inherently more sensitive test for clustering, because the radio surveys contain - at the same flux level - intrinsically faint nearby sources as well as powerful distant sources, and this tends to smear out any genuine spatial clustering.

Since irregularities in the QSO density are likely to reflect non-uniformities in the overall matter density*, these studies should help to test the correctness of the 'hierarchical cosmology' recently discussed by de Vaucouleurs (1970). (Provided that the density within the 'super-super-clusters' were still far below the density of an Einstein-de Sitter universe, all parts of the universe would partake in undecelerated expansion, and no significant deviations from the Hubble law would necessarily occur. Nor, if the density were low enough, would there be any effects on the angular distribution of primordial background radiation. Thus QSOs provide the only firm hope of discovering - or ruling out - a hierarchy of inhomogeneities extending up to the scale of the Hubble radius).

A further, and perhaps less hopeful, possibility is that absorption features in QSO spectra may yield information about the distribution of galaxies or clusters along the line of sight. Several QSOs display complex absorption spectra involving lines with several different redshifts (generally smaller than the redshift derived from the emission lines). Although it now seems more likely that the absorbing material is ejected from the QSO itself, some statistical tests of the alternative viewpoint are discussed by Bahcall and Peebles (1969) and by Roeder (1969).

* If the cosmological evolution of QSOs found by Schmidt (1968) is related to the ambient matter density, then the density of QSOs would vary as (density) ${ }^{3}$. The QSO concentration would then be a very sensitive indicator of overall density inhomogeneities. 


\section{Conclusions}

The main conclusion to emerge so far is that, if the Universe resembles a standard Friedman model, the density of powerful sources must vary with cosmic epoch. This result follows from the radio data alone, but is strengthened by optical studies of QSOs, especially if the redshifts of these objects indicate their distance. The QSO redshift distribution suggests that this remarkably steep evolution (corresponding, in models where $\Lambda=0$, to a source density increase of $10^{2}-10^{3}$ per comoving volume, so that the distance between neighbouring QSOs at $z \simeq 2$ may have been $\sim 30$ times smaller than it is now) continues back to $z \simeq 2.5$. Beyond that point, its $z$-dependence must level off, though there is no firm evidence for any actual decrease at larger redshifts. This view of physical conditions in the early universe blends naturally into the broader scenario of the 'hot big bang' model, which provides the commonly accepted framework for interpreting the 'relict' isotropic microwave background. Observations of QSOs and radio sources promise our most direct hope of studying the interesting epochs when galaxies, and other structures present in the Universe today, were in their early phases of development.

The cause of QSO redshifts is still open to reasonable doubt, and all investigations bearing on this problem should be actively pursued. At present, detailed inferences concerning physical conditions during the epochs $z \simeq(0.3-3)$ - being contingent on two hypotheses: the 'cosmological' interpretation of the redshifts and the assumption of a Friedman model - are something of a 'bootstrapping' operation; and selfconsistency does not guarantee truth. However, even though one would be rash or dogmatic to claim even $50 \%$ likelihood for this evolutionary scheme, it seems more plausible than any specific alternative yet proposed. It is thus sensible to adopt it as a basis for studies of the evolution of QSOs and radio sources, provided that no glaring contradiction emerges.

Unless and until such studies yield a firm understanding of this evolution, it will be impossible to use observations of QSOs and radio galaxies even to determine $q_{0}$ still less to delineate the geometry of the Universe in any greater detail.

\section{Acknowledgements}

Helpful conversations with many colleagues have greatly assisted me in preparing this review, and I would especially like to thank Drs J. G. Bolton, M. S. Longair, M. Schmidt, and J. R. Shakeshaft.

Note added in proof: Since this review was prepared, further evidence has been presented concerning the association of QSOs with other objects. The net effect of this new evidence, however, is to leave the true nature of QSO redshifts as unclear as ever. J. E. Gunn (Astrophys. J. Letters 164, L113, 1971) finds that a galaxy close to PKS $2251+11$ appears to have the same redshift $(z=0.32)$. Unlike B264, PKS $2251+11$ is a 'bona fide' QSO (being substantially brighter than any galaxy). On the 
other hand, H. C. Arp has found a 'bridge' apparently connecting Markarian 205 with NGC 4319, and a similar bridge converting NGC 7603 with a compact companion with larger redshift (Astrophys. Letters 7, 221, 1971). Also, evidence has been presented for a statistically significant association between QSSs in the 3CR catalogue and nearby galaxies (E. M. Burbidge; G. R. Burbidge; P. Solomon and P. A. Strittmatter, 1971).

\section{References}

Aizu, K., Fujimoto, Y., Hasegawa, H., Kawabata, K., and Taketani, M.: 1964, Prog. Theor. Phys. Osaka. Suppl. Ser. 31, 35.

Arp, H. C.: 1970a, Astrophys. Letters 5, 257.

Arp, H. C.: 1970b, Astron. J. 75, 1.

Audretsch, J. and Dehnen, H.: 1969, Astron. Astrophys. 3, 252.

Bahcall, J. N.: 1969, Astrophys. J. 158, L87.

Bahcall, J. N. and Peebles, P. J. E.: 1969, Astrophys. J. Letters 156, L7.

Bahcall, J. N., Schmidt, M., and Gunn, J. E.: 1969, Astrophys. J. Letters 157, 177.

Bahcall, N. A. and Bahcall, J. N.: 1970, Publ. Astron. Soc. Pacific 82, 1276.

Bailey, J. A. and Pooley, G. G., 1968, Monthly Notices Roy. Astron. Soc. 138, 57.

Bardeen, J. M.: 1970, Nature 226, 64.

Barnothy, J. M. and Barnothy, M. F.: 1968, Science 162, 348.

Bash, F. N.: 1968, Astrophys. J. 152, 375.

Bolton, J. G.: 1969, Astron. J. 74, 131.

Bolton, J. G., Gardner, F. F., and Mackey, M. B.: 1964, Australian J. Phys. 17, 340.

Braccesi, A., Ceccarelli, M., Fanti, R., and Giovannini, C.: 1966, Nuovo Cimento 41B, 92.

Braccesi, A. and Formiggini, L.: 1969, Astron. Astrophys. 3, 364.

Braccesi, A., Lynds, C. R., and Sandage, A. R.: 1968, Astrophys. J. Letters 152, L105.

Bridle, A. H.: 1967, Monthly Notices Roy. Astron. Soc. 136, 219.

Burbidge, G. R.: 1967, Astrophys. J. 147, 851.

Burbidge, G. R.: 1968, Astrophys. J. Letters 154, L41.

Burbidge, G. R. and Burbidge, E. M.: 1969, Nature 224, 21.

Christiansen, W.: 1969, Monthly Notices Roy. Astron. Soc. 145, 327.

Cowan, C. L.: 1968, Astrophys. J. Letters 154, L5.

Davidson, W.: 1962a, Monthly Notices Roy. Astron. Soc. 123, 425.

Davidson, W.: 1962b, Monthly Notices Roy. Astron. Soc. 124, 79.

de Vaucouleurs, G.: 1970, Science 167, 1203.

De Young, D. S. and Axford, W. I.: 1967, Nature 216, 129.

Doroshkevich, A. G., Longair, M. S., and Zeldovich, Y. B.: 1970, Monthly Notices Roy. Astron. Soc. $147,139$.

Field, G. B.: 1970, in A. and M. Sandage (eds.), Stars and Stellar Systems 9, in press.

Fomalont, E. B.: 1968, Bull. Astron. Inst. Netherl. 20, 69.

Galt, J. A. and Kennedy, J. E. D.: 1968, Astron. J. 73, 135.

Gower, J. F. R.: 1966, Monthly Notices Roy. Astron. Soc. 133, 151.

Grueff, G. and Vigotti, M.: 1968, Astrophys. Letters 2, 113.

Harris, B. J. and Kraus, J. D.: 1970, Nature 227, 785.

Heeschen, D. S.: 1966, Astrophys. J. 146, 517.

Holden, D. J.: 1966, Monthly Notices Roy. Astron. Soc. 133, 225.

Hoyle, F.: 1968, Proc. Roy. Soc. London, Ser. A. 308, 1.

Hoyle, F., Burbidge, G. R., and Sargent, W. L. W.: 1966, Nature 209, 751.

Hoyle, F. and Fowler, W. A.: 1967, Nature 213, 373.

Hughes, R. G. and Longair, M. S.: 1967, Monthly Notices Roy. Astron. Soc. 135, 131.

Jauncey, D. L.: 1967, Nature 216, 877.

Kafka, P.: 1967, Nature 213, 346.

Kardashev, N. S.: 1967, Astrophys. J. Letters 150, L135.

Kellermann, K. I., Pauliny-Toth, I. I. K., and Davis, M. M.: 1968, Astrophys. Letters 2, 105.

Legg, T. H.: 1970, Nature 226, 65. 
Lequeux, J.: 1970, paper presented at conference on 'Radio Sources and the Universe', Groningen, April 1970.

Longair, M. S.: 1966, Monthly Notices Roy. Astron. Soc. 133, 421.

Longair, M. S. and Pooley, G. G.: 1969, Monthly Notices Roy. Astron. Soc. 145, 121.

Longair, M. S. and Scheuer, P. A. G.: 1967, Nature 215, 919.

Longair, M. S. and Scheuer, P. A. G.: 1970, Monthly Notices Roy. Astron. Soc. 151, 45.

Lynden-Bell, D.: 1969, Nature 223, 690.

Lynds, C. R. and Wills, D.: 1970, Nature 226, 532.

Macleod, J. M., Swenson, G. W., Yang, K. S., and Dickel, J. R.: 1965, Astron. J. 70, 756.

McVittie, G. C. and Stabell, R.: 1967, Astrophys. J. Letters 150, L141.

Miley, G. K.: 1968, Nature 218, 933.

Mills, D. M. and Sturrock, P. A.: 1970, Astrophys. Letters 5, 105.

Oort, J. H.: 1961, O.E.C.D. Symposium on 'Large Antennae for Radio Astronomy', Paris.

Pacholczyk, A. G. and Weymann, R. J.: 1968, Astron. J. 73, 836.

Pauliny-Toth, I. I. K., Kellermann, K. I., and Davis, M. M.: 1971, this volume, p. 444.

Peach, J. V.: 1971, this volume, p. 314.

Petrosian, V. and Ekers, R. D.: 1969, Nature 224, 484.

Petrosian, V. and Salpeter, E. E.: 1968, Astrophys. J. 151, 411.

Petrosian, V., Salpeter, E. E., and Szekeres, P.: 1967, Astrophys. J. 147, 1222.

Piper, D. E.: 1968, Nature 219, 846.

Plagemann, S. H., Feldman, P. A., and Gribbin, J. R.: 1969, Nature 224, 875.

Pooley, G. G.: 1968, Nature 218, 153.

Pooley, G. G. and Ryle, M.: 1968, Monthly Notices Roy. Astron. Soc. 145, 211.

Rees, M. J. and Schmidt, M.: 1971, Monthly Notices Roy. Astron. Soc., in press.

Rees, M. J. and Sciama, D. W.: 1967, Nature 213, 374.

Rees, M. J. and Setti, G.: 1968, Nature 219, 127.

Refsdal, S.: 1969, Astrophys. J. 155, 373.

Roeder, R. C.: 1969, Astrophys. J. Letters 157, L153.

Rowan-Robinson, M.: 1968a, Monthly Notices Roy. Astron. Soc. 138, 445

Rowan-Robinson, M.: 1968b, Monthly Notices Roy. Astron. Soc. 141, 445.

Rowan-Robinson, M.: 1969, Nature 224, 1094.

Ryle, M.: 1968, Ann. Rev. Astron. Astrophys. 6, 249.

Ryle, M. and Longair, M. S.: 1967, Monthly Notices Roy. Astron. Soc. 136, 123.

Ryle, M. and Neville, A. C.: 1962, Monthly Notices Roy. Astron. Soc. 125, 39.

Sandage, A. R. and Luyten, W. J.: 1967, Astrophys. J. 148, 767.

Sandage, A. R. and Luyten, W. J.: 1969, Astrophys. J. 155, 913.

Sandage, A. R. and Miller, W.: 1966, Astrophys. J. 144, 1238.

Scheuer, P. A. G.: 1970, in A. and M. Sandage (eds.), Stars and Stellar Systems 9, in press.

Schmidt, M.: 1966, Astrophys. J. 146, 7.

Schmidt, M.: 1968, Astrophys. J. 151, 393.

Schmidt, M.: 1969, Ann. Rev. Astron. Astrophys. 7, 527.

Schmidt, M.: 1970, in D. O. 'Connell (ed.), Pont. Acad. Sci. Scripta Varia, 35, Vatican City, in press.

Shakeshaft, J. R.: 1968, in R. R. Daniel, P. J. Lavakare and S. Ramadurai (eds.), Cosmic Ray Studies, Tata Institute, Bombay.

Shimmins, A. J., Bolton, J. G., and Wall, J. V.: 1968, Nature 217, 818.

Shklovsky, I. S.: 1967, Astrophys. J. Letters 150, L1.

Solheim, J. E.: 1968a, Nature 217, 145.

Solheim, J. E.: 1968b, Nature 219, 415.

Strittmatter, P. A., Faulkner, J., and Walmsley, M.: 1966, Nature 212, 141.

Sturrock, P. A. and Feldman, P. A.: 1968, Astrophys. J. Letters 152, L39.

Terrell, J.: 1966, Science 154, 1282.

Terrell, J.: 1968, Phys. Rev. Letters 21, 637.

van der Laan, H.: 1969, Bull. Astron. Inst. Neth. 20, 171.

Véron, P.: 1966, Nature 211, 724.

Wall, J. V.: 1970, Ph.D. Thesis, Austr. Nat. Univ., unpublished.

Weedman, D. W.: 1970, Astrophys. J. Letters 161, L113. 
Wilson, A. G.: 1967, Mem. Soc. Roy. Sci. Liege 23, 125.

Woltjer, L.: 1971, this volume, p. 277.

\section{Discussion}

Tovmasjan: Recently Dr Arakelian at Byurakan Observatory made a count of identified quasi-stellar radio sources. The result is that the slope of the $\log N-\log S$ relation is -1.5 if the optical luminosities were considered. The slope is appreciably higher when radio fluxes were considered. From these data he concluded that the evolution of radio luminosities rather than of their space density is taking place. He came to the same conclusion applying Schmidt's $V / V_{m}$ test separately to the radio and optical data. For optical data the mean value of $V / V_{m}$ is 0.5 and is larger when radio limits were considered.

Ozernoy: Recently Dibai and Zasov at Sternberg Institute summarized the absolute optical magnitudes of the nuclei and integrated magnitudes of Seyfert galaxies. One can obtain from their data that the optical luminosity of the nucleus is proportional to the square of the luminosity of the whole galaxy. Just the same relation is discovered by I. Pronik of the Crimean Observatory for radioluminosity of nuclei and optical luminosity of normal spirals. It follows from these correlations that the non-thermal emission is nearly constant during the entire active phase because the optical luminosity of a galaxy is obviously constant. This is evidence for density evolution rather than luminosity evolution.

Miley: The intrinsic dispersion in the radio luminosity of quasars is much larger than the intrinsic dispersion of linear separation and for the steeper spectra sources the angular diameter is a far better 'standard candle' than is the flux density. Would you regard number counts of angular diameters as a useful additional cosmological test?

Rees: In principle, yes. However, any subdivision of the radio sources introduces selection effects, as well as making the statistics worse than for the whole sample. There may also be some evolutionary dependence of linear sizes on redshifts, but I think there is more chance of our being able to estimate this eventually than of our being able to understand the compact phases of radio sources.

King: Regarding the optical search for quasars, this can be done by photographic photometry in UBV. Both the excess in U - B and the variability give you a chance to find quasars with good completeness down to a faint limit.

Wagoner: Since Dave Jauncey is not here, I would like you to comment on the desirability of using differential number-flux relations rather than integral number-flux relations as a way of reducing the effects of measurement errors.

Rees: Of course it is helpful to plot the counts differentially if one wants to estimate the formal errors in the slope, since individual points on the $N(S)$ curve are then independent. Also, there is an important respect in which integral counts may even be misleading, which is the following. Suppose that the integral count can be fitted by two straight lines of different slopes, joining at a flux density $S_{0}$. One might then think that something happens to the counts at $S_{0}$. However, if one were to plot the differential counts, they would be found to start to curve at a flux density higher than $S_{0}$. Thus, if one looks only at the observed integral counts (which are steep at high $S$ and flatter at low $S$ ) one might be tempted to overestimate the extent, and the significance, of the region of steep slope.

Godart: The stagnation period in Lemaitre's Universe produces a very slight effect to form preferentially galaxies, anyway on the basis of statistical fluctuations. In the case of a short stagnation period, the galaxies will not yet be formed now. In the case of a long stagnation period approaching a static universe, the accumulation of quasar redshifts, around $z=2$, will not be extremely conspicuous. The speeding-up of the formation of galaxies needs the application of a new physical theory. It is felt that it must be non-equilibrium theory.

Miss Merkelijn: The results of the volume-halves $\left(V / V_{m}\right)$ test for radio galaxies is very similar to that for QSOs. Wouldn't this weaken the argument that the result for QSOs is partly due to a selection effect in the redshift determination, as the two classes of objects are not likely to be affected by the same selection effect? Also, I wonder whether the fact that for the radio galaxies the same result obtains as for QSOs does give us some information about whether QSO redshifts are also cosmological.

Kafka: I should like to point out a difficulty which one has to be aware of, if one uses the 'luminosityvolume test' or similar procedures. In order to count objects of certain kinds in spheres around us, 
one must determine for each object its 'range of visibility', within which it would appear in the complete sample. Thus the completeness may not be sufficiently defined by some limits of observed brightness. For instance, an individual range, ' $V_{m}$ ', of visibility may contain gaps between us and the object. This happens as a rule in world models with visible antipole(s), but it can happen also due to spectral features like steep low-frequency cutoffs or lines. For the $3 \mathrm{C}$ sample used by Schmidt there seem to be no important corrections of this type, but with future samples one should be careful. Especially, the fact that the difficulty of measuring the redshift changes considerably as lines are shifted into or out of the visible spectrum, may produce gaps in $V_{m}$, and the corresponding selection effects can be eliminated only if a refined definition of completeness is used in the computation of $V_{m}$. 